\title{
OS DESAFIOS DA ANAMNESE E EXAME FÍSICO NA SISTEMATIZAÇÃO DA ASSISTÊNCIA DE ENFERMAGEM -SAE: REVISÃO INTEGRATIVA DE LITERATURA
}

\author{
THE CHALLENGES OF ANAMNESIS AND PHYSICAL EXAMINATION IN THE \\ SYSTEMATIZATION OF NURSING CARE -SAE: AN INTEGRATIVE LITERATURE \\ REVIEW
}

\author{
Andressa Melo de Moraes ${ }^{1}$ \\ Deize Viana Vasconcelos ${ }^{2}$ \\ Thaianna Cristina Oliveira Imbiriba ${ }^{3}$
}

RESUMO: INTRODUÇÃO: O presente estudo tem como objetivo investigar os desafios da anamnese e exame físico na sistematização da assistência de enfermagem - SAE do município de Monte Alegre - PA. Trata se de um estudo descritivo com abordagem do tipo qualitativos que busca investigar os desafios da anamnese e exame físico na sistematização da assistência de enfermagem. A anamnese e o exame físico são etapas do processo de enfermagem, apresentando como instrumento de grande valor para a assistência de enfermagem. Portanto, o processo de enfermagem apresenta desafios para estabelecimento de todas as suas fases. OBJETIVO: $O$ presente estudo tem como objetivo investigar os desafios da anamnese e exame físico na Sistematização da Assistência de Enfermagem. MATERIAIS E MÉTODOS: Trata-se de um estudo interativo, descritiva de abordagem qualitativa. Seguiu a coleta de dados em etapas, estabelecimento da questão da pesquisa, busca na literatura, categorização dos estudos, interpretação dos resultados e apresentação da revisão realizadas nas bases de artigos em plataforma digital. RESULTADOS E DISCURSÕES: O desenvolvimento das etapas do processo de enfermagem vem enfrentando dificuldades, as quais enfermeiros e instituições procuram superar. Portanto há estratégias que podem ser aproveitadas pelos profissionais enfermeiros e instituições de saúde para a implementação e consolidação na prática profissional. Estas estratégias destacam a implementação e desenvolvimento de ações em educação permanente, informatização da assistência, e apoio das instituições de ensino superior. CONCLUSÃO: Diante disso, o processo de enfermagem, permite organizar e planejar as ações de enfermagem a partir da tomada de decisão do enfermeiro.

\footnotetext{
I Acadêmica do curso de Bacharelo de Enfermagem, IO $^{\circ}$ semestre noturno, do Centro Universitário da Amazônia-UNAMA, Santarém-PA, Brasil.E-mail: Andressamelooos@gmail.com.

${ }^{2}$ Acadêmico do curso de Bacharelo de Enfermagem, Io $^{\circ}$ semestre noturno, do Centro Universitário da Amazônia-UNAMA, Santarém-PA, Brasil.E-mail: deizeviana86@gmail.com.

${ }^{3}$ Docente do curso de Enfermagem do Centro Universitário da Amazônia UNAMA, enfermeira Especialista em Saúde Coletiva. E-mail: thaianna.imbiriba@hotmail.com.
} 
Palavra Chave: Avaliação. Implementação. Planejamento.

ABSTRACT: INTRODUCTION: This study aims to investigate the challenges of anamnesis and physical examination in the systematization of nursing care - SAE in the city of Monte Alegre - PA. This is a descriptive study with a qualitative approach that seeks to investigate the challenges ofanamnesis and physical examination in the systematization of nursing care. Anamnesis and physical examination are steps in the nursing process, presenting them as a valuable instrument for nursing care. Therefore, the nursing process presents challenges for the establishment of all its phases. OBJECTIVE: This study aims to investigate the challenges of anamnesis and physical examination in the Systematization of Nursing Care. MATERIALS AND METHODS: This is an interactive, descriptive study with a qualitative approach. Data collection followed in stages, establishment of the research question, literature search, categorization of studies, interpretation of results and presentation of the review carried out in the databases of articles on a digital platform. RESULTS AND DISCOURSIONS: The development of the stages of the nursing process has been facing difficulties, which nurses and institutions try to overcome. Therefore, there are strategies that can be used by professional nurses and health institutions for the implementation and consolidation of professional practice. These strategies highlight the implementation and development of actions in continuing education, computerization of care, and support from higher education institutions. CONCLUSION: In light of this, the nursing process allows for the organization and planning of nursing actions based on the nurse's decision-making process.

Keyword: Assessment. Implementation. Planning.

\section{INTRODUÇÃO}

Conforme a Universidade Federal do Paraná (2014), o desenvolvimento da Sistematização da Assistência de Enfermagem (SAE) nos serviços de Saúde converge com preceitos ético-legais da profissão do Enfermeiro previstos na Lei do Exercício Profissional 7498/86, do Código de Ética da Profissão, assim como as Resoluções 272/2002 e 358/2009 do Conselho Federal de Enfermagem, que defini a Sistematização da Assistência de Enfermagem em que os serviços de Saúde dispõe da implementação do Processo de enfermagem em ambientes, públicos ou privados.

Ainda nesta linha de estudo o autor indaga que, a anamnese e o exame físico são elementos de grande importância para o processo de enfermagem, pois trata-se de uma metodologia sistematizada que o profissional enfermeiro dispõe para a prestação de cuidados humanizados na assistência. Sua aplicação possibilita determinar e monitorar 
problemas de responsabilidade dos enfermeiros e simultaneamente auxilia os demais profissionais da equipe de enfermagem a avaliarem os diagnósticos de enfermagem, prevenindo complicações contribuindo com a melhora na qualidade da assistência prestada.

Segundo Meire (2019), o processo de Enfermagem é composto por cinco fases que se inter-relacionam dinamicamente: investigação, diagnósticos de enfermagem (DE), planejamento, implementação e avaliação da assistência de enfermagem. Quando o enfermeiro está apto a definir diagnósticos de enfermagem, estabelecer prescrições e avaliar a evolução do paciente, ele torna-se capaz de planejar a alta do seu cliente juntamente com sua equipe multiprofissional.

A anamnese, é a primeira etapa do processo de assistência, fase crucial para que se obtenham, através da entrevista, dados históricos para identificar problemas e determinar diagnósticos. O exame físico, busca investigar sinais e sintomas através de interpretação observando pontos relevantes e anormalidades conforme as técnicas de inspeção, palpação, percussão e ausculta (SANTOS; VEIGA; ANDRADE, 20II).

Duarte et al (2015), relata que o risco de queda de pacientes no leito é um dos eventos adversos que necessita ser criteriosamente analisado, pois o mesmo pode causar ferimentos e sequelas aos pacientes, prolongando o tempo da internação hospitalar. Outra problemática muito discutida que a literatura destaca, é a ocorrência de eventos adversos relacionados à integridade cutânea do paciente, devido a não realização das mudanças de decúbito, trocas de curativos, além de, posicionamento inadequado no leito, por consequência o surge o desenvolvimento de úlceras por pressão em pacientes acamado.

Como base desta mesma linha de pensamento o mesmo autor enfatiza a responsabilidade dos profissionais de enfermagem em comunicar e registrar por escrito todas as suas ações de modo correto, e confiável, conforme estabelecido pelo Código de Ética Profissional de Enfermagem. Os princípios éticos devem ser seguidos por todos os profissionais como a beneficência, veracidade, justiça, competência e fidelidade, que sustentam os esforços para uma prática segura e de respeito aos direitos dos pacientes. 
Acrescenta-se ainda que, quanto maior a demanda de pacientes, maior é a necessidade para se planejar a assistência, já que a sistematização das ações visa o planejamento e a organização com eficiência e veracidade da assistência prestada. Dessa forma, a implantação do sistema da Sistematização de assistência de enfermagem (SAE), atualmente, é considerado um desafio, principalmente, no que diz respeito à gerência da assistência, uma vez que em uma realidade complexa, e multidimensional, exige do enfermeiro empenho e criatividade na construção e execução de estratégias inovadoras e participativas (SOARES et al., 2015).

Diante dessas questões, houve a necessidade de analisar qual a importância da anamnese e do exame físico na assistência de enfermagem, propondo uma avaliação dos principais fatores que afetam a qualidade na assistência buscando a qualidade no atendimento e satisfação do cliente. Dessa forma, com embasamento nos fatos citados esse estudo será de base para todos os profissionais da área de saúde com o objetivo de avaliar os desafios em planejar, acompanhar, avaliar o cliente e realizar diagnósticos de enfermagem e sugerir mudanças que possam contribuir com a qualidade da assistência prestada pela equipe de saúde aos usuários.

\section{FUNDAMENTAÇÃO TEÓRICA}

\section{I Sistematização da Assistência de Enfermagem (Sae)}

A Sistematização da Assistência de Enfermagem (SAE) trata-se de uma metodologia de organização, planejamento e execução de ações sistematizadas de enfermagem, realizadas pela equipe no decorrer do tempo em que o indivíduo se encontra na assistência de enfermagem. (SANTOS et al., 2014).

Nesta mesma linha de pensamento o autor destaca que, conforme a resolução 359/2009 a Sistematização de Enfermagem é constituída de cinco etapas: Histórico de Enfermagem, Diagnóstico de Enfermagem, Planejamento de Enfermagem, Implementação e Avaliação de Enfermagem, tendo como objetivo identificar as necessidades do paciente apresentando uma proposta ao seu atendimento e cuidado, direcionando a Equipe de Enfermagem nas ações a serem realizadas. 
Segundo Sousa et al (2020), a estrutura da Sistematização da Assistência de Enfermagem (SAE) sustenta-se como base a forma de tratar o sujeito, por meio de um olhar holístico, efetivando o princípio da integralidade, assim como as necessidades biológicas, emocionais, psicológicas, sociais e espirituais. Seguindo a mesma linha de pensamento, o autor indaga que a Sistematização da Assistência de Enfermagem é um instrumento de avaliação, fiscalização do cuidado e auditoria, sendo de grande importância para a gestão, por avaliar a qualidade da assistência e garantir a veracidade dos procedimentos realizados durante o período de hospitalização.

Conforme Meire (2019), as necessidades humanas básicas estão relacionadas com a sobrevivência física, psíquica e espiritual, quando essas necessidades não são supridas totalmente, ocasiona um desconforto para o ser humano, que com o tempo pode afetar a enfermidade do mesmo, dessa forma, devem ser realizadas em virtude do próprio bemestar do paciente, quando essas necessidades surgem, a assistência do profissional de enfermagem habilitado faz-se necessário.

Esse modelo assistencial vem sendo desenvolvido ao longo do tempo por enfermeiros comprometidos no cuidado prestado ao paciente, pois compreendem a necessidade do cuidado interativo, complementar e multiprofissional. Apesar disso, observa-se que para a efetivação da Sistematização da Assistência de Enfermagem (SAE), a equipe de enfermagem precisa de uma atuação com eficácia para obter um atendimento humanizado e adequado para os pacientes, tendo inúmeros desafios na sua implantação e nos serviços de saúde (SANTOS et al., 2014).

O uso dos Diagnósticos de Enfermagem direciona a assistência de enfermagem para as necessidades de cada indivíduo, facilitando a escolha da conduta mais adequada para o cliente, aos cuidados da enfermagem. Os estudos apontam melhorias na adoção da SAE, assim como a preconização do Diagnóstico de Enfermagem. (CONCEIÇÃO et al., 2017).

Rodrigues et al (202I), descrevem que a Sistematização da Assistência de Enfermagem garante atenção adequada e proporciona melhor qualidade dos cuidados prestados e soluções dos problemas, fortalecendo a qualidade do serviço ofertado 
estimulando uma relação terapêutica. A aplicação da Sistematização da Assistência de Enfermagem (SAE) contribui com a prática diferenciada dos enfermeiros atuando nos níveis de complexidade, buscando promover uma reintegração das pessoas em sofrimento psíquico, além de proporcionar aos clientes o bem-estar biopsicossocial. No entanto para que a sistematização se concretize é necessário que haja uma transformação nas relações de interação com a equipe multiprofissional e preparo dos mesmos.

Vale destacar que o diálogo é um passo importante da comunicação através da qual se pode promover a integração e interação, tanto da equipe de enfermagem, quanto do profissional/cliente, para que juntos possam enfrentar as dificuldades impostas, manter ou adotar ações que levam a práticas seguras, melhorias na comunicação, trabalho em equipe e compartilhamento de conhecimentos (CAVALCANTE et al., 2015).

Além do mais é necessário basear-se em conhecimentos científicos, intensificando o pensamento crítico e o raciocínio clínico. Conhecimentos e procedimentos teoricamente organizados, sistematizados e sempre reformulados, que se constituem em base segura para a ação eficiente. Visto que essa metodologia permite diagnosticar as necessidades, elaborar as prescrições adequadas e avaliar a evolução do cliente nos cuidados de enfermagem (BARROS; LOPES, 2010).

Soares et al (2015), destaca que a estrutura física é fundamental para a operacionalização da SAE. Já que, uma sala privativa para troca de informações sobre o plantão, torna-se um espaço para os profissionais se expressarem espontaneamente, colaborando para definir as ações de enfermagem que se colocarão em prática por meio da SAE, dentro do local de trabalho.

Oliveira e Curado (2019), evidenciam em seu estudo com base nos conhecimentos e habilidades do profissional Enfermeiro(a) com relação às taxonomias de enfermagem conhecidas pelos usuários, $57,8 \%$ afirmam conhecer alguma taxonomia, mas não souberam recordar dos títulos delas. E, dentre as descritas, destacaram-se NANDA-I, NIC, NOC e a Classificação Internacional para a Prática da Enfermagem (CIPE). Já II,4\% (n=68) declararam não conhecer nenhuma taxonomia. 
Para Barreto et al (2020), existem diferentes fatores que interferem na aplicação da SAE nas instituições hospitalares pesquisadas. Percebeu-se a desmotivação com os colegas e o local de trabalho como fatores determinantes para a não realização da SAE, a falta de tempo dos enfermeiros, atribuído, principalmente, à sobrecarga de trabalho e ao número reduzido de profissionais e a grande demanda de pacientes na unidade hospitalar, prejudica a sua aplicabilidade.

Conforme Silva et al (2019), são muitas as vantagens que esse processo significa, infelizmente sua implantação é um desafio visto que, a insegurança no desempenho de funções dentro da sistematização é resultado da falta de conhecimento de seus próprios deveres dificultando assim a qualidade da assistência ofertada. Seguindo a lógica do autor, uma realidade complexa, multifacetada e multidimensional, exige do enfermeiro empenho e criatividade na elaboração e execução de estratégias inovadoras e participativas.

\subsection{O papel do enfermeiro para a implementação da SAE}

Florence Nightingale, afirmava que a enfermagem requeria conhecimentos distintos daquelas da medicina, definindo as premissas em que a profissão deveria ser fundamentada em conhecimento sobre a enfermagem direcionado às pessoas, e às condições em que elas viviam e em como o ambiente poderia atuar, de forma positiva ou negativa (OLIVEIRA; CURADO, 2019).

De acordo com Carnauba (2016), as instituições de saúde de todo o Brasil iniciaram a adoção das teorias de enfermagem como pressupostos básicos sob os quais os cuidados de enfermagem deveriam ser realizados, ampliando o foco do cuidado para além das questões biológicas, e também, as dimensões sociais, psíquica e espiritual, a forma do olhar holístico para o doente com o objetivo de melhoria na qualidade da assistência de enfermagem.

Conforme Menezes et al (2011), a competência do enfermeiro caracteriza-se pela abrangência de sua atuação e compreensão de suas responsabilidades e deveres perante a equipe e seus pacientes, é necessário assumir um compromisso com sua aplicação na prática e a responsabilidade em executar suas etapas, aprimorando e atualizando seus conhecimentos. Muito mais do que competência técnica, é necessário que os enfermeiros 
tenham sensibilidade de captar as necessidades emergentes, capacidades para empreender e estimular ações inovadoras e, especialmente, obter conhecimento, e estratégias para que possa envolver e comprometer aos demais profissionais da equipe de saúde.

Segundo Nanda (202I), enfatiza a importância a respeito do raciocínio clínico frente a assistência de enfermagem, pois é um processo, informado e reformada por novos dados ou evidências além de também ser iterativo, quanto mais informações adquirimos, mais informações somos capazes de sintetizar, desvendando o problema e descobrindo a formação de padrões.

O instrumento primordial do trabalho da enfermagem é o cuidar, este é o ponto principal do seu desenvolvimento, pois possui um alcance muito além do atendimento às necessidades básicas do ser humano. Tem o poder de alcançar o indivíduo no desenvolvimento da sua autonomia, independência, autoestima e autocuidado (MEDEIROS et al. apud SOARES, 2017).

De acordo com Lourenco (2020), os cuidados de enfermagem tem como foco o planejamento, a organização e avaliação de atenção a promoção dos projetos de saúde de cada cidadão, independentemente da sua raça, credo ou género, prevenindo doenças, promovendo o processo de readaptação, e satisfação das necessidades humanas fundamentais e a máxima independência na realização das atividades de vida, buscando a adaptação aos défices aos múltiplos fatores.

Seguindo ainda nesta mesma linha de pensamento, o profissional enfermeiro é o elo essencial para o cuidado continuado, pois requer uma preocupação acrescida na sua promoção, prevenção e identificação da problemática acometida, na prestação de cuidados, a defender a privacidade e a intimidade da pessoa doente ou com restrições de qualquer ordem, seja ao nível dos cuidados primários, diferenciados ou continuados, adquirindo conhecimento para o agir profissional, repassando a sua equipe e aos demais profissionais de saúde, segurança na tomada de decisões relacionadas ao paciente.

A falta de compromisso com os planejamentos de metas do trabalho; a falta de censo critico, a disputa de níveis hierárquicos entre os integrantes, a baixa remuneração, os conflitos em diferentes níveis hierárquicos, a falta de conhecimento e habilidade para 
exercer as atividades são alguns dos principais fatores que dificultavam as inter-relações entre os mesmos no seu local de trabalho (SILVA; TEXEIRA; DRAGANOV, 2018).

Dessa forma Albano e Freitas (2013), destacam que, para o desempenho das funções gerenciais é de grande importância que haja um planejamento, ou seja, uma determinação, para que os objetivos sejam alcançados e a definição de como atingi-los da melhor forma possível. A participação do enfermeiro é muito significativa para a organização, pois garanti a assistência com maior eficiência, e efetividade, tendo em vista que o mesmo gerencia a assistência como um todo, refletindo na quantidade e qualidade assistencial.

De acordo com Cruz et al (2019), o perfil desejável do profissional enfermeiro relacionadas à tomada de decisão, incluem: o gerenciamento de dados e documentações clínicas e administrativa dos planos de cuidados, com uso de sistemas de apoio à decisão de protocolos clínicos, ser comunicativo, além da interação com o fluxo de trabalho clínico desenvolvendo conhecimentos, habilidades e atitudes que o prepara, para condução das equipes que devem trabalhar ao alcance dos objetivos em comum de forma efetiva, qualificando sua prática em assumir a posição de liderança e, a apropriação de metodologias que a sustente.

Seguindo a lógica do autor, ao liderar, o enfermeiro tem a chance de construir relações de confiança, estimular a participação ativa no planejamento, e desenvolvimento da avaliação das propostas, permitindo que todos possam agir durante o processo de produção da assistência ao paciente.

Oliveira et al (2012), destacam que o gerenciamento das atividades de enfermagem consiste, direta ou indiretamente, na supervisão e direcionamento das ações que favoreçam o atendimento das necessidades humanas básicas dos pacientes. A falta de uma organização de assistência gera dificuldades, ocasionando perda de um tempo significativo no processo de gerenciamento e desorganização do serviço, bem como a não utilização de uma metodologia de assistência.

Para Silva e Camelo (2013), a liderança é considerada uma das principais competências a ser desenvolvida pelo enfermeiro devendo ser conquistada e aprimorada. Todavia, para que isso aconteça, os enfermeiros necessitam mobilizar algumas 
características capazes de auxiliá-los nesta função, fazendo com que exerça uma liderança íntegra, coerente e ética.

\subsection{Os desafios da enfermagem na efetivação da sistematização da assistência de enfermagem -SAE}

A Enfermagem é uma profissão fundamental na estrutura das profissões de saúde, que busca a longos anos por melhores condições de trabalho e emprego, por salários dignos, por uma jornada de trabalho condizente com a atividade que desempenha e por uma formação emancipadora e de qualidade (FALCÃO, 2020).

Pesquisas enfatizam que, para trabalhar com o Sistema Único de Saúde (SUS), os profissionais de saúde precisam ser qualificados por capacitores que estejam envolvidos no processo de implantação, reorganização do sistema, que tem sem dúvida impulsionado o processo de universalização e descentralização do acesso, instruindo e sensibilizando-os sobre a importância de ter um sistema on-line, para que este seja sustentado adequadamente. Os profissionais precisam ser orientados sobre os novos conceitos constantemente, a respeito das terminologias, funcionalidades e suporte do sistema, de maneira que se sintam preparados para utilização e manuseio das novas tecnologias (ARAUJO et al, 2019).

Conforme Trindade et al (2016), na prática assistencial, há dificuldades na aplicação do processo de enfermagem, e até a não aplicabilidade dele. Observa-se em algumas situações que o processo de enfermagem não é desenvolvido em todas as suas etapas, devido à mecânica dos afazeres rotineiros, à demanda de trabalho, à falta de apoio institucional, em consequência de um número insuficientes de profissionais da saúde, ocasionando à sobrecarga de trabalho, e assim as ações de enfermagem permanecem centradas no senso comum.

Sousa et al (2020), em seu estudo afirma que o acúmulo de atividades, referindo-se a diversas situações vivenciadas, as quais os mesmos consideraram situações-limite a serem enfrentadas, além de funções administrativas e burocráticas e o gerenciamento de conflitos 
na equipe de saúde são alguns dos desafios da Enfermagem na efetivação da Sistematização da Assistência de Enfermagem -SAE.

Com relação a Saúde Coletiva, além de problemas estruturais e de organização dos serviços, da gestão da Estratégia Saúde da Família (ESF) e a prática profissional de suas equipes, padecem de um problema sistêmico incompleto, por falta de oferta de ações e de cuidados de saúde, apesar da disponibilidade de padrões de referência, diretrizes, metas e protocolos de trabalho (FACCHINI et al, 2018).

Na comunidade ou no interior os desafios para implementação da SAE enfrentados pelo profissional enfermeiro se torna mais árduo pois, as populações que convivem nos territórios rurais possuem crenças que diferem das cultivadas em zonas urbanas, ao modo de tratar às doenças, e compreendê-las conforme as terapêuticas adotadas. Diante deste quadro, é fundamental entender que essas situações não podem ser tratadas com práticas lineares, desenvolvidas para perfis epidemiológicos de áreas urbanas, as populações das zonas rurais exigem condições diferentes, relevando-se a competência cultural para efetivar a atenção qualificada (FROTA et al, 2020).

Oliveira et al (2018), em seu estudo afirma que a enfermagem permanece com intensa carga horária de trabalho em relação às demais profissões de saúde de nível superior, a desigualdade aumentou ao longo dos anos em todas as regiões, percebe-se que as demais profissões da saúde obtiveram conquistas em relação à jornada de trabalho.

Esta problemática aumenta quando se interpõem outros obstáculos, como por exemplo, deficiência de materiais e equipamentos, ruídos de comunicação, desgaste físico e emocional dos trabalhadores, além de, limitações da equipe médica na compreensão do trabalho de enfermagem (MARTINS; DALL'AGNOL, 2016).

Outro aspecto importante com relação no que se diz respeito ao gerenciamento e à adequação de horas de trabalho de enfermagem e de pacientes/leito por profissional, é a sobrecarrega dos profissionais de enfermagem o que de fato compromete a capacidade laboral dos mesmos e, com isso, a humanização por eles praticada. Esta sobrecarga, imposta pelo cotidiano do trabalho, resulta na assistência mecanizada e tecnicista, não reflexiva (LIMA et al, 2018). 
A proposta de condições dignas de trabalho e satisfação profissional são desafios constantes, de modo a promover condições e contratos de trabalho adequados com a ética e com o exercício profissional, para poder garantir a equidade de gênero, etnia e cor, desenvolvendo formas de mecanismos de auscultação dos profissionais da saúde, a fim de compreender seus níveis de satisfação e estabelecer estratégias que possam melhorar o desempenho e motivação profissional (THUMÉ et al,2018).

O desenvolvimento da nossa linguagem científica é um processo contínuo, não tem fim ou ponto em que a terminologia estará completa, ao contrário disso, sempre haverá frequentes revisões recentes e acréscimos à terminologia, e retiradas do que já existiu, tendo em vista que o conhecimento insensatamente evolui, algumas dessas evoluções são de natureza editorial, outros são mais complexos e requerem discussões de forma mais extensa, com pesquisas, e análises aprimoradas, para compreender melhor a terminologia NANDA-I tornando a linguagem mais forte dos diagnósticos de enfermagem padronizados e baseados em evidências (NANDA, 202I).

\section{METODOS}

A atual pesquisa trata-se de um estudo de revisão integrativa da literatura de caráter descritivo com abordagem bibliográfica do tipo qualitativo que busca investigar os desafios da Anamnese e Exame físico na Sistematização da Assistência de Enfermagem - SAE.

Atualmente a pesquisa é proveniente por meio de referências bibliográficas promovendo uma das melhores maneiras de iniciar um estudo, analisando semelhanças e diferenças através de artigos levantados nos documentos de referências.

Para Ercole e Alcoforado (2014), a revisão integrativa de literatura tem como finalidade sintetizar resultados obtidos em pesquisas sobre a temática abordada, de maneira sistemática, ordenada e abrangente. A mesma é qualificada integrativa que fornece informações amplas sobre o assunto ou problema, fornecendo conhecimento.

Manzato et al (2012) ressalta que, a pesquisa descritiva, analisa, observa, registra e compara fatos ou fenômenos variáveis sem manipulá-los. Além disso, busca entender as situações e relações que o acontece na vida social, política, econômica e demais aspectos do 
comportamento humano, do indivíduo isoladamente e de grupos e comunidades mais complexas, e cujo registro não consta de documentos.

Para Sousa et al (2021), a pesquisa bibliográfica é uma fase do processo fundamental em todo trabalho científico que dominará todas as etapas de uma pesquisa, consiste no levantamento, seleção, e organização de informações conforme à pesquisa, desenvolvida com base em materiais elaborados, composto principalmente, por livros e artigos científicos.

Segundo Galvão et al (2017), os estudos com métodos qualitativos oferecem explicações aprofundadas de acontecimentos complexos, envolvendo seus aspectos contextuais, ou enfatizam em pesquisas otimizadas envolvendo poucos indivíduos. Assim sendo, seus resultado não são propagados.

A revisão da literatura executada anteriormente possibilitou aos pesquisadores a aquisição de conhecimento, que conduziu o percurso na procura de resolução de um problema. O acervo de informações em meios eletrônicos é um importante progresso para os pesquisadores, visto que esse acesso proporciona uma atualização frequente.

Para coleta de dados foi realizado um levantamento de informações relacionados ao objeto de estudo através artigos em bases de dados: Scientific Electronic Library Online (SciELO), Google Acadêmico, Ministério da Saúde, Revista Brasileira de Enfermagem (REBEn), Literatura Latino-Americana e do Caribe em Ciências da Saúde (LILACS). A estratégia utilizada para localizar os artigos foi por intermédio da palavra-chave "Assistência de Enfermagem" ou da combinação dos seguintes descritores e suas combinações nas línguas portuguesa: "Anamnese", "Exame físico", "Implementação em saúde", "Qualidade da assistência".

Os critérios de inclusão determinado para a seleção dos artigos foram: artigos publicados em português; artigos na sua totalidade que apresentassem a temática referente à revisão integrativa e artigos publicados e indexados nos referidos bancos de dados nos últimos dez anos.

Os critérios de inclusão adotados para orientar a busca e seleção das publicações foram: artigos publicados em periódicos científicos nacionais revisados por pares que 
abordem a temática constante no banco de dados: Scientific Electronic Library Online (SciELO), Google Acadêmico, Ministério da Saúde, Revista Brasileira de Enfermagem (REBEn), Literatura Latino-Americana e do Caribe em Ciências da Saúde (LILACS).

Foram incluídos artigos publicados entre o período de 2010 a 2021, que comtemplassem o estudo em questão e o público-alvo, com relação aos desafios da anamnese e exame físico na Sistematização da Assistência de Enfermagem - SAE visando discutir o tema abordado.

Os critérios de exclusão foram: As publicações que não se encontravam disponíveis em texto completo; as publicações que apresentavam disponibilidade de texto completo, mas cujo link apresentava erro mediante a tentativa de acessá-lo e os artigos fora do período de 2010 a 2021.

Para guiar a revisão integrativa, formulou-se a seguinte questão norteadora: Quais os desafios da anamnese e exame físico na assistência de enfermagem?

A coleta de dados dos artigos se deu entre os meses de maio a novembro de 2021 e foi utilizada uma listagem dos artigos encontrados com título do artigo, autores e ano de publicação. A amostra final desta revisão integrativa foi constituída por 95 noventa e cinco artigos, onde, foram selecionados 37 trinta e sete para exposição.

A apresentação e discussão dos resultados encontrados foi feita de forma descritiva, possibilitando ao leitor a avaliação da aplicabilidade da revisão integrativa elaborada, de forma a atingir o objetivo desse método, ou seja, impactar positivamente na qualidade da prática de enfermagem na Assistência de Enfermagem, fornecendo subsídios para equipe em sua tomada de decisão.

\section{RESULTADO E DISCURSÃO}

$\mathrm{Na}$ presente revisão integrativa foram analisados quarenta e um artigos, que estavam de acordo com os critérios de inclusão pré-estabelecidos. Após uma leitura minuciosa dos artigos selecionados, foram criadas 3 categorias capazes de sinalizar as evidencias cientificas disponíveis nas literaturas sobre Os desafios no papel do enfermeiro para implementação da SAE. 


\section{CONSIDERAÇÕES FINAIS}

O Processo da Sistematização da Assistência de Enfermagem é essencial para o profissional enfermeiro dentro do seu local de trabalho, sendo uma atividade privativa, pois promove uma análise criteriosa $\mathrm{da}$ qualidade $\mathrm{da}$ assistência prestada ao paciente/cliente, e sua aplicabilidade deve ser cumprida, conforme os termos da legislação profissional sendo uma exigência legal.

Observou-se através do estudo que são muitos os fatores que dificultam a implantação da Sistematização da Assistência de Enfermagem, como por exemplo, a sobrecarga de trabalho devido à falta de apoio institucional em contratar mais profissionais da saúde para atender a grande demanda, em consequência disso, a falta de conhecimento teórico sobre o processo de enfermagem por motivos da falta de tempo e motivação.

De acordo com os dados obtidos, evidenciou-se que há inúmeras dificuldades consideradas desafios a serem enfrentados para a Implantação da Sistematização de Enfermagem: a falta de conhecimento sobre a realização do exame físico, dificuldades de identificar o diagnóstico de enfermagem, dificuldade de elaborar a prescrição de enfermagem.

Outro fator importante e essencial foi constatar, que a pratica do cuidado individualizado pautados no conhecimento técnico - cientifico resulta na qualidade da assistência.

Com base nas informações exploradas segundo os artigos analisados, pode-se entender que são muitos os desafios do processo de enfermagem com relação a sua implantação, e é fundamental que a equipe de enfermagem esteja devidamente preparada, perante o ponto de vista da capacitação de conhecimento científico e da habilidade em praticá-la em meio a tantos fatores que impedem o desempenho desse método.

Portanto, é fundamental que os profissionais enfermeiros estejam desenvolvendo uma reflexão junto a equipe de enfermagem, incluindo os demais profissionais de saúde dentro do seu setor em que trabalha, na tentativa de conscientizar o real papel da equipe multidisciplinar, assimilando a importância do método da assistência prestada, cabendo- 
lhes a responsabilidade e competência em assegurar a vida do seu paciente, e com isso colocá-lo em prática.

Segundo os dados da literatura estudada, espera-se contribuir com a equipe de enfermagem, acadêmicos, e enfermeiros atuantes de forma geral, a respeito da temática abordada, auxiliando como fonte de informação para realização de novas pesquisas sobre o tema abordado.

\section{REFERÊNCIAS}

ALBANO, T. C.; FREITAS, J. B. Participação efetiva do enfermeiro no planejamento: foco nos custos. Revista Brasileira de Enfermagem, v. 66, n. 3, p. 372-377, jun. 2013. Disponível em: 〈https://doi.org/10.1590/Soo34-71672013000300oII $>$. Acesso em Io Set. 2021.

ARAÚJO, J. R. et al. Sistema e-SUS AB: Percepções dos enfermeiros da Estratégia Saúde da Família. Revista Saúde em Debate, v. 43, n. 122, p. 780-792, set. 2019. Disponível em: $<$ https://doi.org/ro.159o/oro3-IIo4201912210. Acesso em io Set. 2021.

BARRETO, M. S. et al. Sistematização da assistência de enfermagem: a práxis do enfermeiro de hospital de pequeno porte. Escola Anna Nery - Revista de Enfermagem. v. 24, n. 4, 2020. Disponível em: https://doi.org/10.1590/2177-9465-EAN-2020-0005. Acesso em Io Set 2021.

BARROS, A. L. B. L.; LOPES, J. L. A legislação e a sistematização da assistência de enfermagem. Revista Oficial do Conselho de Enfermagem, v. I, n. 2, p. 63-65, 2010. Disponível em: 〈http://revista.cofen.gov.br/index.php/enfermagem/article/view/r7 >. Acesso em: Io ago. 2021.

CARNAUBA. F. P. Enfermagem e ciência KLS. 2016. Editora e Distribuidora Educacional S.A. Disponível em: 〈http://www.santaisabel.com.br/upl/pagina_adicional/Download__ENFERMAGEM_E_CIENCIA-or-09-2019_I8-5I-I4.pdf $>$. Acesso em ro Set 2021.

CAVAlCANTE, A. K. C. B. et al. Cuidado seguro ao paciente: contribuições da enfermagem. Revista Cubana de Enfermería, v. 31, n. 4, 22 dez. 2015. Disponível em: $\langle$ http://revenfermeria.sld.cu/index.php/enf/article/view/907/r4I〉. Acesso em: io set. 2021.

CONCEIÇÃO, V. M. et al. Sistematização da Assistência de Enfermagem: Uma revisão integrativa. Revista Eletrônica Gestão e Saúde. v. 8, n. 3, p. 395-413, 29 set. 2017. Disponível em: https://periodicos.unb.br/index.php/rgs/article/view/IozIo. Acesso em: II ago. 2021. 
CRUZ, J. R.; GONÇALVES, L. S.; GIACOMO, A. P. M. A. Metodologia ágil Scrum: uso pelo enfermeiro em jogo educativo sobre manejo seguro de medicamentos. Revista Gaúcha de Enfermagem, v. 40, n. spe, 2019. Disponível em: https://doi.org/10.1590/1983I447.2019.20180302. Acesso em: 20 set. 2021.

DUARTE, S. C. M. et al. Eventos adversos e segurança na assistência de enfermagem. Revista Brasileira de Enfermagem, v. 68, n. I, p. 144-154, fev. 2015. Disponível em: 〈http://www.scielo.br/scielo.php?script=sci_arttext\&pid=So034$71672015000100144 \& \operatorname{lng}=$ en $\& n r m=$ iso $>$. Acesso em $20 \mathrm{~J} \mathrm{Jn}$. 2021.

ERCOLE, F. F.; MELO, L. S; ALCOFORADO, C. L. G. C. Revisão integrativa versus revisão sistemática. Reme: Revista Mineira de Enfermagem, v. I8, n. I, 2014. Disponivel em: http://www.dx.doi.org/10.5935/1415-2762.201400or. Acesso: I3 ago. 2021.

FACCHINI, L. A.; TOMASI, E.; DILÉliO, A. S. Qualidade da Atenção Primária à Saúde no Brasil: avanços, desafios e perspectivas. Revista Saúde em Debate, v. 42, n. sper, p. 208-223, set. 2018. Disponível em: https://doi.org/10.1590/o103-I1042018Sir4. Acesso em 21 Set. 2021.

FALCÃO, V. T. F. L. Os Desafios da enfermagem no enfrentamento a COVID-I9. Revista Enfermagem Digital e Cuidado e Promoção da Saúde, v. 5, n. I, 2020. Disponível em: http://www.dx.doi.org/I0.5935/2446-5682.202000or. Acesso em: 2I Set. 2021.

FROTA, M. A. et al. Mapeando a formação do enfermeiro no Brasil: desafios para atuação em cenários complexos e globalizados. Ciência \& Saúde Coletiva, v. 25, n. I, p. 25-35, jan. 2020. Disponível em: https://doi.org/10.1590/1413-81232020251.27672019. Acesso em 21 Set. 2021.

GAlVAO, M. C. B.; PlUYE, P.; RICARTE, I. L. M. Métodos de pesquisa mistos e revisões de literatura mistas: conceitos, construção e critérios de avaliação. InCID: Revista de Ciência da Informação e Documentação, v. 8, n. 2, p. 4-24, 4 out. 2017. Disponível em: https://www.revistas.usp.br/incid/article/view/r21879. Acesso em: 20 de Jan de 2021.

LIMA, A. A.; JESUS, D. S. D.; SILVA, T. L. Densidade tecnológica e o cuidado humanizado em enfermagem: a realidade de dois serviços de saúde. Physis: Revista de Saúde Coletiva, v. 28, n. 3, 20 dez. 2018. Disponível em: https://doi.org/10.1590/Soro373312018280320 . Acesso em: 20 de Jan de 2021.

LOURENÇO, H. O papel do enfermeiro na sexualidade do cidadão com problemas no seu “continuum de saúde." Revista Portuguesa de Enfermagem de Saúde Mental, n. 23, jun. 2020. Disponível em <http://scielo.pt/scielo.php?script=sci_arttext\&pid=SI6472160202000010000I\&lng=pt\&nrm=iso $>$. Acesso em Io set. 2021. 
MANZATO, A.; ADRIANA, P.; SANTOS, B. A Elaboração de questionários na pesquisa quantitativa. Departamento de Ciência de Computação e Estatística IBILCE - UNES . 2012 Disponível em: 〈http://www.inf.ufsc.br/ vera.carmo/Ensino_2012_I/ELABORACAO_QUESTIONARI OS_PESQUISA_QUANTITATIVA.pdf>. Acesso em ro Set 202I.

MARTINS, F. Z.; DALL'AGNOL, C. M. Centro Cirúrgico: desafios e estratégias do enfermeiro nas atividades gerenciais. Revista Gaúcha de Enfermagem, v. 37, n. 4, 2016. Disponível em: https://doi.org/ro.1590/1983-I447.2016.04.56945. Acesso em: 21 Setembro 2021.

MEDEIROS, B. D. F. S. et al. O Papel do nfermeiro ao idoso acamado em instituição de longa permanência. Revista Conexão Eletrônica - Três Lagoas, MS - Volume I4 Número I $\quad-\quad$ Ano 2017. Disponível em: https://www.mprj.mp.br/documents/20184/542936/30_O_PAPEL_DO_PAPEL_DO_ENF ERMEIRO_AO_IDOSO_ACAMADO_EM_INSTITUICAO_DE_LONGA_PERMANE NCIA.pdf. Acesso em: ir de julho de 202I.

MEIRE C. T.; MARIA, A. SAE: Sistematização da Assistência de Enfermagem: guia pratico. $3^{\underline{a}}$ ed. Rio De Janeiro: Guanabara Koogan, 2019.

MENEZES, S. R. T.; PRIEL, M. R.; PEREIRA, L. L. Autonomia e vulnerabilidade do enfermeiro na prática da sistematização da assistência de enfermagem. Revista da Escola de Enfermagem da USP, v. 45, n. 4, p. 953-958, ago. 2011. Disponível em:< https://doi.org/ro.1590/Soo8o-62342011000400023 >. Acesso: em 8 Jan. 2021.

NANDA. International Nursing Diagnoses: Definitions \& Classification, Thieme Medical Publishers. 2021-2023.

OLIVEIRA, B. L. C. A.; SILVA, A. M.; LIMA, S. F. Carga semanal de trabalho para enfermeiros no Brasil: Desafios ao exercício da profissão. Trabalho, Educação e Saúde, v. I6, n. 3, p. 1221-1236, 2 ago. 2018. Disponível em: https://doi.org/10.1590/1981-7746-solooi59. Acesso em: Acessado 2I Setembro 202I.

OLIVEIRA, C. M. et al. Percepção da equipe de enfermagem sobre a implementação do processo de enfermagem em uma unidade de um hospital universitário. Revista Mineira de Enfermagem, v. $16, \quad$ n. 2, p. 258-263, 2012. Disponivel em: http://www.reme.org.br/artigo/detalhes/527. Acesso em: 21 Set. 2021.

OLIVEIRA, M.; CURADO, A. C. C. Enfermagem, ciência e trabalho - Londrina: Editora e Distribuidora Educacional S.A., 2019. Disponivel em: http://www.santaisabel.com.br/upl/pagina_adicional/Download_- 
_ENFERMAGEM_CIENCIA_E_TRABALHO-04-09-2019_20-34-53.pdf. Acesso em: 2i Set. 202I.

RODRIGUES, T. T. et al. A Sistematização da assistência de enfermagem: um década de implementação sob a ótica do enfermeiro. Revista Enfermagem Atual In Derme, v. $95, \quad \mathrm{n}$. 34, 13 abr. 2021. Disponível em: https://revistaenfermagematual.com/index.php/revista/article/view/996. Acesso em: I3 de ago. 2021.

SANTOS, N.; VEIGA, P.; ANDRADE, R. Importância da anamnese e do exame físico para o cuidado do enfermeiro. Revista Brasileira de Enfermagem, v. 64, n. 2, p. 355-358, abr. 20II. Disponível em: http://www.scielo.br/scielo.php?script=sci_arttext\&nrm=iso\&lng=pt\&tlng=pt\&pid=Soo3 4-7167201100020002i\#end. Acesso em: 21 de Set. 2021.

SANTOS, W. N. et al. Sistematização da assistência de enfermagem: o contexto histórico, o processo e obstáculos da implantação. JMPHC | Journal of Management \& Primary Health Care ISSN 2179-6750, v. 5, n. 2, p. 153-158, 17 jul. 2014. Disponível em: https://www.jmphc.com.br/jmphc/article/view/210. Acesso em: 10 ago. 2021.

SIlVA, F. M. L.; CARVALHO, J. J. M.; ALMEIDA, L. C. P. Dificuldades na implementação da Sistematização da Assistência de Enfermagem na Unidade de Terapia Intensiva Adulto. Revista Eletrônica Acervo Saúde, n. 28, p. e986, i8 jul. 2019. Disponivel em: https://doi.org/ro.25248/reas.e986.2019. Acesso em: 2I de setembro de 2021.

SILVA, M. M.; TEIXEIRA, N. L.; DRAGANOV, P. B. Desafios do enfermeiro no gerenciamento de conflitos entre a equipe de enfermagem. Revista de Administração em Saúde, v. I8, n. 73, 28 dez. 2018. Disponivel em: http://dx.doi.org/10.23973/ras.73.138. Acesso em: 28 de out de 202I.

SILVA, V. L. S.; CAMELO, S. H. H. A competência da liderança em enfermagem: conceitos, atributos essenciais e o papel do enfermeiro líder. Rev. enferm. UERJ, p. 533-539, 2013. Disponível em: https://pesquisa.bvsalud.org/portal/resource/pt/bde-27859. Acesso em: og fev. 202I.

SOARES, M. et al. Sistematização da assistência de enfermagem: facilidades e desafios do enfermeiro na gerência da assistência Systematization of nursing care: challenges and features to nurses in the care management Sistematización de los cuidados de enfermería: facilidades y desafíos de enfermeros en el soporte de gestión PESQUISA | RESEARCH. Escola Anna Nery - Revista de Enfermagem, v. 19, n. I, p. 47-53, 2015. Disponível em: https://doi.org/10.5935/1414-8145.20150007. Acesso: em 20 Jan. 2021. 
SOUSA, A. S.; OLIVEIRA, S. O.; ALVES, L H. A pesquisa bibliográfica: princípios e fundamentos. Cadernos da Fucamp, v.20, n.43, p.64-83/2021. Disponível em: http://www.fucamp.edu.br/editora/index.php/cadernos/search/authors. Acesso em: I3 ago. 202I.

SOUSA, B. V. N. et al. Benefícios e limitações da sistematização da assistência de enfermagem na gestão em saúde / Benefits and restrictions of systematization of nursing assistance in health management. Journal of Nursing and Health, v. Io, n. 2, 3I mar. 2020. Disponível

em: https://periodicos.ufpel.edu.br/ojs2/index.php/enfermagem/article/view/15083/III83. Acesso em: 20 ago. 2021.

THUMÉ, E. et al. Formação e prática de enfermeiros para a Atenção Primária à Saúde avanços, desafios e estratégias para fortalecimento do Sistema Único de Saúde. Revista Saúde em Debate, v. 42, n. sper, p. 275-288, set. 2018. Disponível em: https://doi.org/ro.1590/o103-II042018Sir8. Acesso em: 21 Set. 2021.

TRINDADE, L. R. et al. Processo de enfermagem: desafios e estratégias para sua implementação sob a ótica de enfermeiros. Revista Saúde (Santa Maria), v. 42, n. I, p. 75, 30 jun. 2016. Disponível em: https://doi.org/ro.5902/2236583419805. Acesso em: 20 ago. 2021.

UNIVERSIDADE FEDERAL DO PARANÁ. Hospital de Clínicas, Diretoria de Enfermagem-Comissão de Sistematização da Assistência de Enfermagem (COMISAE). Avaliação de enfermagem: anamnese e exame físico (adulto, criança e gestante) Curitiba: Hospital de Clínicas, 20I4. Disponivel em: https://docero.com.br/doc/nvines. Acesso em: I3 ago. 2021. 